\title{
New Insights into the Molecular Mechanisms of Podocytes Injury in Diabetes
}

\author{
Irina Bobkova* , Natalia Chebotareva, Anna Schukina, Elena Kamyshova, and Larisa Bobrova
}

Department of Rheumatology, Nephrology and Occupational Diseases, I.M. Sechenov First Moscow State Medical University (Sechenov University), Moscow, Russia

${ }^{*}$ Corresponding author: Irina Bobkova, Department of Rheumatology, Nephrology and Occupational Diseases, I.M. Sechenov First Moscow State Medical University (Sechenov University), Moscow, Russia, Tel: +7917-559-71-43; E-mail: irbo.mma@mail.ru

Received date: September 11, 2018; Accepted date: September 24, 2018; Published date: September 28, 2018

Citation: Bobkova I, Chebotareva N, Schukina A, Kamyshova E, Bobrova L (2018) New Insights into the Molecular Mechanisms of Podocytes Injury in Diabetes. J Clin Exp Nephrol Vol.3 No.3: 17. DOI: 10.21767/2472-5056.100068

Copyright: @2018 Bobkova I, et al. This is an open-access article distributed under the terms of the Creative Commons Attribution License, which permits unrestricted use, distribution, and reproduction in any medium, provided the original author and source are credited.

\section{Abstract}

Pandemia of diabetes mellitus (DM) remains one of the biggest worldwide medical problems associated with dangerous complications, particularly diabetic nephropathy (DN), which is the leading cause of the end-stage renal disease. It was confirmed convincingly today that podocytes injury is implicated primarily or secondarily in various proteinuric glomerular diseases, including DN. The characteristic signs of podocytopathy are podocytes hypertrophy, foot process effacement, detachment from the glomerular basal membrane and apoptosis. Structural and functional alterations of podocytes evolve early, outpacing clinically significant albuminuria. The increasing loss of podocytes is associated with morphological and clinical signs of DN progression.

This review details the molecular and cellular events, mediators and signaling pathways that contribute to podocytes injury in DM, discusses the most important achievements, clinical and experimental data in these issues, describes the urinary biomarkers of podocytes injury.

Keywords: Diabetic nephropathy; Podocytes injury; Podocytes effacement; Proteinuria; Podocytopenia; Glomerulosclerosis; Urinary biomarkers

\section{Abbreviations:}

DM: Diabetes Mellitus, DN: Diabetic Nephropathy, AU: Albuminuria, PU: Proteinuria, MAU: Microalbuminuria, NAU: Normoalbuminuria, T1DM: Type1 Diabetes Mellitus, T2DM: Type2 Diabetes Mellitus, FP: Foot Process, GBM: Glomerular Basal Membrane, SD: Slit Diaphragm, GFR: Glomerular Filtration Rate, RAAS: Renin-Angiotensin-Aldosterone-System, AT-II: Angiotensin II, AT1R: AT-II type 1 receptors, PPR: Peceptor for propenin, AGEs: Advanced Glycation End Products, RAGE: Receptor for AGE, EMT: Epithelial-mesenchymal transitition, TGF- $\beta 1$ : Transforming Growth Factor $\beta 1$, VEGF: Vascular Endothelial Growth Factor, VEGFR: Vascular Endothelial Growth Factor Receptor, ROS: Reactive Oxygen Species, MAPK: Mitogen- activated Protein Kinase, TRPC: Transient Receptor Potential Canonical Channel, NADPH: Nicotinamide Adenine Dinucleotide Phosphate, NOX: NADPH Oxidases, mTOR: Mammalian Target of Rapamycin, MMP-9: Matrix Metalloproteinase 9, NFkB: Nuclear Factor Kappa-light-chain-enhancer of Activated B cells, WT1: Wilms Tumor Protein 1, ELISA: Enzymelinked Immunosorbent Assay

\section{Introduction}

Till today pandemia of diabetes mellitus (DM) remains one of the biggest worldwide medical problems. 425 million people in the World have DM, there will be 629 million in 2045 [1]. One of the greatest dangers of DM is diabetic nephropathy (DN) that develops in $30-40 \%$ of diabetic patients and often leads to the end-stage renal disease $[2,3]$.

The various cell types are involved in the kidney damage including glomerular endotheliocytes [4], mesangiocytes [5], podocytes [6] and tubular epitheliocytes [7]-all of them are the targets of hyperglycemic injury. Several decades ago scientific interest was focused on the mesangium proliferation, mesangial matrix expansion as a cellular and molecular basis of DN ("mesangiocentric" DN concept) [8]. However, this DN theory did not explain clearly the origin of proteinuria (PU), especially massive. While a full understanding of the PU mechanisms in DN remains to be achieved, emerging studies have emphasized the crucial role of podocytes in these processes [9-15].

It was confirmed by the morphological data, that podocytes changes are primarily or secondarily implicated in various proteinuric glomerular diseases $[16,17]$. Moreover, there are evidences of podocytes depletion associated with the glomerulosclerosis in chronic progressive glomerulopathies, including DN [9]. Therefore, podocytes dysfunction and loss, their relationship with metabolic and hemodynamic disorders have attracted scientific attention today in order to find new methods of early DN diagnostic and prevention of disease progression.

In this article the molecular mechanisms of podocytes injury in DN are discussed, the most important achievements in these issues, clinical and experimental data are reviewed. 


\section{Podocytes Pathobiology in DN}

The unique podocyte structure provides a wide range of its functions and the adaptive responses in physiological conditions, but at the same time makes this cell very sensitive to injury. Under the influence of various pathogenic factors (toxic, metabolic, hemodynamic) structural and functional changes occur in podocytes $[9,10,15,18,19]$. The characteristic features of podocytes injury (podocytopathy) are the foot process (FP) widening and effacement, podocytes hypertrophy, apoptosis, detachment from the glomerular basal membrane (GBM) and loss into the urinary space (podocyturia) and podocytes number reduction in the glomeruli (podocytopenia).

The diagnosis of podocytopathy can be carried out by morpho-pathological examination of kidney biopsy material (light and electron microscopy, immunohistochemical identification of podocytes specific proteins) or by the detection and quantification of urinary biomarkers (Figure 1).

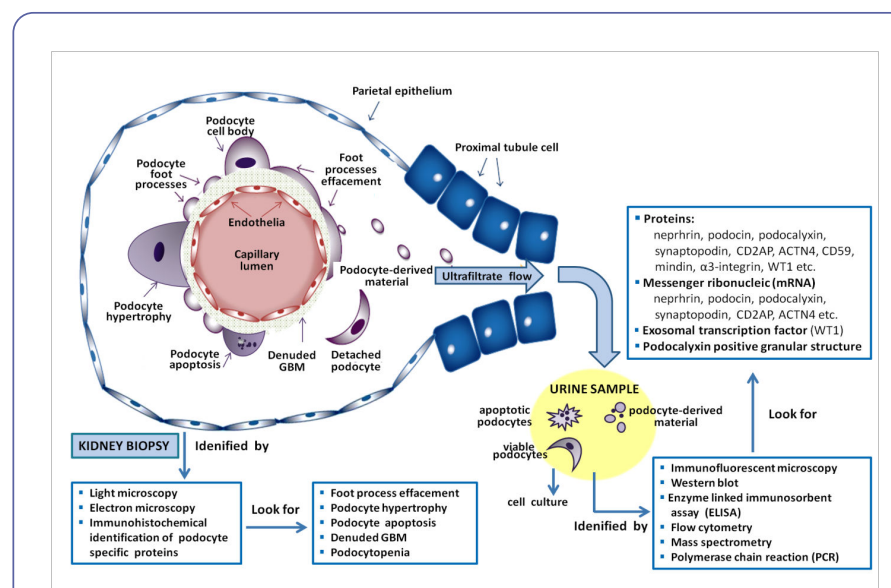

Figure 1: Characteristic signs of podocytopathy and methods of podocytes injury detection.

\section{Podocytes effacement}

Podocytes can undergo a process termed effacement, where the normal cellular architecture of the FP is altered and results in flattening and spreading of the FP along the GBM and disruption of the slit diaphragm (SD). The effacement can manifest whenever the following occurs: i) the disruption of SD and its related protein-protein interactions; ii) the interference in the normal association of the FP with the underlying GBM; iii) the reorganization of the actin cytoskeleton and its related protein-protein interactions; iv) the disruption of the normal negatively charged apical surface of the FP [9]. The change in the podocyte shape is not simply a passive process following injury; it is the result of complex molecular interactions of different protein domains of podocytes [17].

The FP widening and effacement in type 1 (T1DM) and 2 DM (T2DM) has been confirmed by a number of experimental and clinical studies, and a direct correlation has been established between the severity of these changes and the albuminuria (AU)/PU degree $[6,10,20,21]$.
The mechanisms of podocytes actin cytoskeleton damage in DN are still being studied. The role of improper calcium handling due to abnormal activation of the calcium TRPC6 and 5 (transient receptor potential canonical) channels, dysregulation of Rho GTPases activity and violated RhoA signaling in podocytes is widely discussed [22-24].

\section{The slit diaphragm, the role of nephrin}

The unique barrier, that regulates passage of macromolecules from the blood to the urine, is the SD, which represents the contacts between podocytes. Several proteins (nephrin, CD2AP, ZO-1, FAT, nephrin-like1, podocin, P-cadherin) determine the SD structure $[14,25,26]$.

Transmembrane protein nephrin functions not only as the core component of the extracellular SD filtration network but also as a signaling scaffold via interactions at its short intracellular region [26]. The extracellular nephrin domain connects with nephrin molecules from the neighboring FP. The cytoplasmic nephrin domain is phosphorylated on tyrosine residues, that is critical to the regulation of various intracellular podocytes pathways, including the actin cytoskeleton remodeling, the PI3 kinase/Akt signal pathway activation, the calcium signaling and nephrin endocytosis [22,27-29]. Nephrin colocalizes with adherence junction proteins such as P-cadherin or FAT, and anchors lipid rafts to the filtration slit. The interaction with CD2AP connects nephrin to the actin cytoskeleton. Nephrin binding to podocin provides the function in cell signaling $[26,30]$.

As it was shown by previous morphological studies in animal models and in humans, the impairment of nephrin expression in the SD was observed not only in congenital nephrotic syndrome with a NPHS1 gene mutation, but also in various acquired nephropathies [31-34]. The different studies of kidney biopsies in patients with DN revealed the decreased glomerular expression of nephrin and the interrelation of these changes with the disorder of podocytes FP [35-37].

It is discussed that focal defects of the SD due to nephrin dissociation from podocytes actin cytoskeleton with following nephrin separation and its excretion into urine (nephrinuria), are some of the mechanisms of PU in various nephropathies [38]. Nephrinuria as a marker of podocytes damage was found in DM $[34,37,39]$. In a cross-sectional study Patari $A$, et al. assessed the presence of nephrin fragments in the urine of patients with T1DM by immunoblotting method [39]. Nephrinuria was revealed in $30 \%$ of DM patients with normoalbuminuria (NAU), in $17 \%$ of patients with microalbuminuria (MAU), and in $28 \%$ of patients with PU, whereas it was not detected in healthy individuals. Belinda Jim performed a quantitative estimation of nephrinuria by ELISA method in T2DM patients [37]. Nephrinuria was found in $100 \%$ of diabetic patients with MAU and PU, as well as in $54 \%$ of patients with NAU.

Relatedly, observation of the FVB/NJ Akita mouse DN model noted that urinary nephrin can be revealed before detectable $\mathrm{AU}$ develops [40]. It was shown that nephrinuria, evaluated by different methods, is closely correlated with the clinical manifestations of DN (MAU/PU, arterial hyprtension, glomerular 
filtration rate (GFR)) [34,37,41-43]. In the work of $\mathrm{Ng} D$. nephrinuria was associated with lower GFR even among normoalbuminuric diabetic patients, who are traditionally perceived as having a low risk of chronic kidney disease [34]. The presented data pointed that nephrinuria is a sensitive indicator of glomerular injury in DM, therefore, it has a good perspectives of application for early diagnostic and monitoring of DN.

\section{Podocyturia}

Glomerular podocytes damage is accompanied by appearance of not only their structural-functional proteins in the urine, but also whole podocytes themselves due to their detachment from GBM [44]. The alterations in the level or activity of adhesive molecules are discussed as a possible mechanism of podocytes detachment. It was shown by the experimental models of DN and in the podocytes cell culture that even the short-term influence of hyperglycemia leads to the reduced expression of $\alpha 3 ß 1$-integrins in podocytes and $\alpha 3 ß 1$-integrin receptors in GBM, to the podocytes detachment from the GBM and their appearance in the urine (podocyturia) [45-47]. Disconnected from GBM podocytes usually die as a result of violations of cellmatrix relationships that are necessary to maintain the podocytes viability. Nevertheless, Vogelmann SU and other investigators were able to culture cells expressing typical podocytes markers from urine, indicating that some podocytes may remain viable [48].

Nakimura T, et al. [49] investigated in T2DM patients the urinary podocytes by immunofluorescence using antibodies against surface protein podocalyxin. The podocyturia was revealed in a half of patients with MAU and in $80 \%$ of patients with PU, furthermore, the number of labeled podocytes in the urine of patients with PU was significantly higher than in patients with MAU. Yu et al. [50] demonstrated that podocyturia was a more sensitive marker of glomerular damage than Hara et al. [51] using the ELISA method detected the podocyturia in a half of diabetic patients with normoalbuminuria, suggesting that the podocytes injury occurres early, prior to MAU appearance. Ye et al. [52] by a flow cytometry method also demonstrated the significantly increased number of podocalyxin-positive podocytes in DM patients with normal AU. The nonparametric ROC analysis confirmed that the sensitivity and specificity of the podocyturia are significantly greater than creatinine clearance, traditionally used for DN assessment.

\section{Urinary biomarkers of podocytes injury, methods of detection}

Whole podocytes themselves (viable as well as apoptotic), podocytes derived proteins, exosomes and granular structures may be detected in the urine of patients with glomerulopathy-all of them may be used as potential urinary tests for podocytes injury (Figure 1). Urinary biomarkers that have been found to be specific for podocytes detection include proteins (nephrin, podocin, synaptopodin, podocalyxin, mindin, Wilms tumor protein 1 (WT1) and etc.), specific messenger ribonucleic acids (mRNAs) (mRNAs of nephrin, podocin, synaptopodin, podocalyxin, CD2-associated protein-CD2AP, an exosomal transcription factor (WT1), and podocalyxin positive granular structures. Different techniques can be used to identify the podocytes derivations, including the immunofluorescent staining, western blot, enzymelinked immunosorbent assay (ELISA), flow cytometry, mass spectrometry, polymerase chain reaction.

The main advantages of urinary diagnostic tests are high specificity, non-invasive detection and reproducibility, availability of measurement by relatively simple and sensitive methods (for instance, ELISA).

Over the past few decades, in addition to described above nephrin, other urinary podocytes biomarker were detected in DM, their associations with the clinical and morphological characteristics of DN were confirmed [41,42,51-56]. The utilization of the podocyte specific urinary biomarkers has not yet translated into common clinical practice, while the age-old quantification of PU, GFR and renal biopsy are the mainstays that clinicians have at hand. But these urinary biomarkers have good prospects for use in the near future as indicators of diabetic glomerulopathy presence/progression and therapeutic efficacy that in some cases may be more advantageous than the established parameters currently used in practice.

\section{Podocytes apoptosis}

Two mechanisms are proposed to explain the podocytes loss: detachment and apoptosis. The increased programmed cell death as a major cause of reduced podocyte number, leading to $\mathrm{PU}$ and glomerulosclerosis was showed in transforming growth factor $\beta 1$ (TGF- $\beta 1$ ) transgenic mice, CD2AP-/- mice and puromycin aminonucleoside (PAN)-treated rats [57-60]. It was discussed that $\sim 90 \%$ of urinary podocytes are apoptotic [9]. The previous data also demonstrated that in DM podocytes are shed from the GBM not as a result of a defect in the podocytes themselves, but in response to some damaging stimulus in the glomerular compartment. Regulation of podocytes survival and death depends on the balance of pro- and antiapoptotic factors [9]. Apoptosis of podocytes is activated by the following factors: hyperglycemia, angiotensin II (AT-II), AT1-receptor, TGF- $\beta 1$, Smad-7, reactive oxygen species (ROS), detachment from the GBM, mechanical tension, reduction of inhibitors of activated cyclic kinases p27 and p21, the basic fibroblast growth factor, apoptosis-inducing factor [9,57-59,61,62]. Antiapoptotic effects have been confirmed for a number of factors, including cyclin I, podocytes proteins (nephrin and CD2AP), intracellular apoptosis inhibitor $\mathrm{Bcl}-2$, preserved cell-cell contacts, vascular endothelial growth factor (VEGF), hepatocytes growth factor, insulin-like factor, heat shock protein 27 and etc., $[9,63]$.

A number of works in vivo and in vitro has already shed light on the delicate molecular mechanisms of podocyte apoptosis induced by leading damaging factors in DM, it will be detailed in the next section.

\section{Autophagic process}

Autophagy is the natural, multi-step process of lysosomal degradation, mediated by the autophagy-related genes and their associated enzymes. It plays a crucial role in removing protein aggregates and damaged or excess organelles, including 
mitochondria, to maintain intracellular homeostasis. Adaptive autophagy may protect renal cells, including podocytes, against cellular stresses under diabetic conditions.

Impairment of podocytes autophagy by up regulation of mTORC1 pathways is implicated in the initiation and progression of DN $[22,64,65]$. Hyperglycaemia stimulates oxidative stress and ROS generation with activation of mTORC1, reduction of MAPK and Sirt1 that leads to autophagy impairment. Thus, improvement of autophagy by mTORC1 inhibitor, or MAPK and Sirt1 activators may be novel therapeutic options in DM, which in addition to RAAS blockers may slow down the DN progression.

\section{Epithelial-mesenchymal transitition (EMT)}

The podocytes loss is facilitated by the activation of EMT [66]. It was shown in a cell culture that under the influence of the main inductor of EMT-TGF- $\beta 1$, podocytes lose ability to express proteins specific for podocytes (nephrine, podocin, P-cadherin, E-cadherin, ZO-1, etc.), they change epithelial phenotype and start to express the markers specific for mesenchymal cells (fibroblast-specific protein-1 (FSP1), desmin, matrix metalloproteinase 9 (MMP-9), a-Smooth muscle actin, etc.) $[67,68]$. As a result of these processes, the podocytes lose the normal cytoskeleton structure, cell polarity, intercellular contacts, and become mobile, resulting in enhanced detachment from GBM and the development of podocyturia. Like fibroblasts, the transdifferentiated podocytes acquire the ability to produce matrix proteins (fibronectin, collagen, etc.), thus speeding up the glomerulosclerosis formation.

In a retrospective cross-sectional analysis, greater amounts of FSP1-positive podocytes were observed in urinary sediments of diabetic patients with MAU than in those with normalbuminuria [69]. FSP1-positive podocytes selectively expressed Snail1, a known trigger for EMT. This study suggests that appearance of FSP1 in podocytes of diabetic patients is associated with more severe clinical and pathological findings of DN and correlates with podocytes detachment due to EMT process.

\section{Podocytes hypertrophy}

Glomerular hypertrophy is present in the early phase of DN. Podocytes in enlarged glomeruli have to cover a larger GBM area and are subjected to increased mechanical stress and injury $[9,70,71]$. Biochemically, hypertrophy is characterized by the entry of podocytes in G1-phase of the cell cycle, accompanied by an increase of protein content in the cells; however, under the certain circumstances, the podocytes stop in G1/S-phase without specific to the $S$-phase increase in DNA synthesis. These intracellular events determine the increase in size (but not numbers) of podocytes. The initial hypertrophy of the damaged podocytes is considered as adaptive. By this way the neighboring epithelial cells try to "patch up" the locuses of denuded GBM. However, after a certain time, the hypertrophy becomes maladaptive because the inducing mechanisms activate the apoptosis of hypertrophied and adjoining podocytes simultaneously. It was demonstrated in vitro and in vivo in diabetes that AT-II induces the podocytes hypertrophy and regulates its intensity by increasing in the synthesis of inhibitors of cyclin-dependent kinases p21 and p27 [71,72].

\section{Podocytopenia}

Due to the limited proliferative capacity of podocytes, a massive podocytes loss leads to reduction in the glomerular podocytes number (podocytopenia). The consequences of different degrees of podocytes loss were demonstrated in rat models of regulated podocytes depletion $[16,72,73]$. Podocytes loss less than $20 \%$ causes the mesangial expansion only. Loss of more than $20 \%$ of podocytes leads to appearance of denuded areas of GBM and formation of synechia with Bowman's capsule. When a glomerulus losses between $20-40 \%$ of its podocytes, it renders itself to segmental sclerosis. The glomerulus becomes progressively sclerotic with more than $40 \%$ podocyte loss. Expressed depletion of more than $60 \%$ of podocytes leads to irreversible defect of the glomerular filter with the persistent high PU and the global glomerulosclerosis.

There are emerging experimental and clinical studies showing that the number of podocytes in the glomeruli is an important determinant of DN progression. Therefore, Steffes MW, et al. [74] in a longitudinal study of patients with T1DM have demonstrated that the decline in glomerular podocytes number is directly correlated with PU increase, and that podocytopenia can develop even in a short duration of DM.Meyer T, et al. [75] revealed in a longitudinal study of the Pima Indian population with T2DM that glomerular podocytes number was the most significant predictor of PU growth and GFR decline. Vestra $M$, et al. [21] showed in European cohort of patients with T2DM that glomerular podocytes pool decreases during progression NAU to PU. In the cross-sectional study of kidney biopsies of patients with T2DM White $\mathrm{K}$, et al. [76] established a significant correlation between $\mathrm{PU}$ and the decrease of podocytes layer density and the reduction of podocytes number in the glomeruli.

\section{Molecular events, mediating the podocytes injury in DM hyperglycemia}

It was shown in murine T1DM and T2DM models that podocytes apoptosis increased sharply with onset of hyperglycemia and coincided with the onset of AU [61]. Elevated extracellular glucose concentration rapidly increased ROS generation via nicotinamide adenine dinucleotide phosphate (NADPH) oxidase and mitochondrial pathways and induced podocytes apptosis by activation of proapoptotic mitogenactivated protein kinase (MAPK) p38 and caspase- 3 cascade [61]. Inhibition of NADPH oxidase activity prevented the podocytes apoptosis and AU [61]. Wang W, et al. showed that hyperglycemia induced mitochondria fission by promoting phosphorylation and lead to consequent generation of mitochondrial ROS [77]. Eid AA, et al. demonstrated that high glucose induced ROS by sequential upregulation of cytochrome p450 and its metabolite 20-hydroxyeicosatetraenoic acid, subsequent increase in the NADPH oxidases (Nox1 and Nox4) expression, and NADPH-dependent superoxide anion generation [78]. It was also shown that treatment of podocytes with named cytochrome p450 metabolite induced podocytes apoptosis similar to the effects of high glucose concentration. At the same 
time inhibition of cytochrome P450A in OVE26 mice (T1DM model) prevented oxidative stress and reduced both FP effacement and apoptosis and significantly decreased AU [78]. It was demonstrated by Liu B.C. that high glucose induced ROS and activated TRPC6 resulting in intracellular $\mathrm{Ca} 2+$ overload and apoptosis [79]. It was reported in other studies that exposure of podocytes to high glucose increased the intracellular $\mathrm{Ca}^{2+}$ concentration and led to calcineurin activation and subsequent nuclear accumulation of nuclear factor of activated T-cells [80], whereas its inhibition ameliorated podocytes injury and DN in $\mathrm{db} / \mathrm{db}$ mice [81].

\section{Insulin signaling}

Podocytes express the insulin receptors and are the main target of insulin action in the glomerulus, exhibiting insulindependent PI3K and MAPK signaling both in vitro and in vivo $[30,82,83]$. There is accumulating evidence that glomerular function is impaired when the podocytes are rendered insulin resistant. In animal models of type 1 and T2DM, glomerular insulin signaling was lost early in the DN progression of diabetic kidney disease [84]. Podocyte-specific deletion of insulin receptor causes $A U$, increased glomerular matrix accumulation, GBM thickening, changes of podocytes morphology and apoptosis [85].

The exposure of podocytes to high glucose abrogates insulin signaling by increasing the molecule Src homology-2 domaincontaining phosphatase-1, which binds to the insulin receptors and prevents downstream signaling [86].

Impaired insulin signaling by altered levels of fatty acids in DM and related metabolic syndrome or proinflammatory cytokines that accumulate during insulin resistance is implicated in podocyte injury $[30,82,83]$. At the same time, the restoring insulin sensitivity and glucose uptake in glomerular podocytes can ameliorate the podocytes injury in DM $[82,87]$.

The insulin pathway is closely associated with the mTOR (mammalian target of rapamycin) signaling in podocytes, which is important for cellular sensing of nutrient and growth factor levels as well as cellular stress [22,30]. Insulin via the insulin receptor and connection with insulin-like growth factor activates mTORC1 through Akt signaling; conversely, mTORC2 inhibits insulin signaling.

Under physiologic conditions, mTOR activity is essential for normal podocyte function. However, in DM elevated mTOR activity also mediates adverse effects, incuding podocytes hypertrophy, apoptosis, impairment of autophagy [22,30,64].

\section{Renin-Angiotensin-Aldosterone System (RAAS)}

Recent studies have shown that podocytes generate many components of the RAAS and express receptors of RAAS, including AT-II, mineralocorticoid and prorenin receptors [88-91]. High glucose concentrations induce AT-II synthesis by podocytes through activation of angiotensinogen expression [6]. Apart from hyperglycemia, the podocytes production of AT-II is activated by ROS, mechanical stretching, and PU components [91-93].
Crucial functions of podocytes, such as contraction, apoptosis, cytoskeletal organization and autophagocytosis, were shown to be regulated by the AT-II type 1 receptors (AT1R) [91,95-97]. Thus, it is believed that AT-II may promote PU via AT1R in podocytes without affecting blood pressure and glomerular hemodynamic changes. There is a large body of evidence that RAAS inhibitors reduce PU by inhibiting the effect of AT-II on podocytes directly $[49,96,98,99]$. So, it was demonstrated that podocytes responded to AT-II with an increase of the intracellular $\mathrm{Ca} 2+$ concentration and activation of $\mathrm{Cl}$ conductance leading to podocytes depolarization. At the same time in rat glomerular in situ and in cell culture the effect of AT-II on $\mathrm{Ca} 2+$ and podocytes depolarization was completely inhibited by low concentrations of the selective AT1R antagonist losartan, suggesting that the effects were mediated by the AT1R [95]. Recently it was shown AT-II contributes to podocytes injury by increasing TRPC6 expression and this pathway is implicated in podocytes injury occurring in DN [23,24,100-102]. AT-II modulates the podocytes dysfunction by suppressing of the nephrin expression [103]. In vitro exposure to stretch induced a decrease of nephrin expression, which was mediated via AT1R [103]. Experimental studies in rat model with streptozotocininduced DM and clinical studies in patients with T2DM showed that treatment with angiotensin-converting enzyme inhibitors or AT1R antagonists restored the nephrin expression and prevented the podocytes loss $[49,98,99,102]$. AT-II via AT1R stimulation increases nephrin- $\beta$-arrestin2 binding, nephrin endocytosis and glomerular permeability in mice [104]. This molecular mechanism of AT-II supports the use of AT1R blockers to prevent $A U$ even in normotensives. AT-II increased VEGF expression in podocytes and AT-II mediated increase of 3 (IV) collagen expression were impared by an inhibitor of VEGF signaling $[106,107]$. AT-II also decreased the expression of heparan sulfate proteoglycans on the cell surface and in the extracellular matrix of podocytes in vitro, suggesting that it might regulate the GBM permselectivity [108].

The expression of the prorenin receptors (PRR) by podocytes has been detected recently $[88,90]$. Under diabetic conditions, elevated plasma prorenin and rennin bind to the PRR in podocytes, resulting in an increased catalytic activity of these proteins and subsequently in local AT-II production [91, 108]. Moreover, prorenin and renin via PRR can activate ATIIindependent intracellular MAPK p38 signaling, leading to podocytes apoptosis and PU $[90,91,109]$. The PRR were shown to be essential for podocytes autophagy and survival [110]. It has been revealed recently, that AT-II promotes autophagy in podocytes by ROS generation and enhanced podocytes expression of autophagic genes such as LC3-2 and beclin-1 [97]. As it was expected, the proautophagic effect of AT-II was inhibited by antioxidants [98]. The PRR have been assumed to be involved in the pathogenesis of DN by the release of cytokines [111]. The renin inhibitor aliskiren inhibited glucoseinduced intracellular AT-II levels and profibrotic and apoptotic effects of high glucose concentrations in podocytes in vitro, although it did not inhibit PRR-dependent extracellular signaling $[112,113]$.

Podocytes express mineralocorticoid receptors, and aldosterone may implement pleiotropic effects via this 
communication [91,114]. Aldosterone is an important player of podocyte injury. Recently it has been shown that aldosteron induces the mitochondrial dysfunction, an increase of ROS and a reduction of the nephrin expression in podocytes [115]. So, the treatment with aldosterone antagonists and prevention of podocytes oxidative stress can be the other ways of podocytopathy correction $[114,115]$.

\section{Transforming growth factor $\beta 1$ (TGF- $\beta 1$ )}

Hyperglycemia, advanced glycation end-products (AGEs), VEGF, PU components (mainly albumin) activate the TGF- $\beta 1$ synthesis by podocytes $[10,57,116]$. Unlike mesangial cells, podocytes do not overexpress TGF- $\beta 1$ in response to AT-II. AT-II increases the podocytes expression of the TGF- $\beta$ type II receptors [39]. Furthermore, AT-II may enhance the ROS generation and, subsequently, oxidative stress activates the latent TGF- $\beta$ and the TGF- $\beta$ signaling system $[117,118]$.

TGF- $\beta 1$ suppresses the glomerular expression of a3b1 integrin that leads to the impaired podocytes adhesiveness to GBM and their detachment $[8,118,119]$.

The significant increase of TGF- $\beta 1$ induces podocytes apoptosis, which promotes podocyturia and podocytopenia. TGF- $\beta 1$ regulates the apoptosis via the Smad3- and Smad7signalling pathways, which in turn inhibit NF-KB, that encodes the synthesis of various cell survival factors $[6,10,57,58]$. It was revealed that TGF- $\beta 1$-induced mitochondrial Nox4 upregulation via the TGF- $\beta 1$ receptor-Smad3 pathway is responsible for ROS production, mitochondrial dysfunction and apoptosis [120]. TGF$\beta 1$ also mediates Smad-independent podocytes apoptosis via MAPK p38 and caspase-3 activation [82,118,119,121]. Recently an alternative theory has been put forward for TGF- $\beta 1$ mediated podocytes injury. TGF- $\beta 1$ increases mitochondrial membrane potential and oxygen consumption rate via mTOR pathway, resulting in increased ROS generation and podocytes apoptosis [122].

Several studies have shown an important role of TGF- $\beta 1$ as a key EMT inductor in various kidney diseases, including DN [66]. TGF- $\beta 1$ induces the desmosomes destruction, cell-matrix remodeling, stress fibers formation $[57,64]$. It was demonstrated that activated TGF- $\beta 1$ signaling in the podocytes may lead to the changes of podocytes phenotype, characterized by reduced expression of SD proteins, increased expression of mesenchymal markers (such as Snail, FSP1, desmin) and overproduction of $\alpha 3(I V)$ collagen and matrix-modifying enzyme MMP-9 [67,116-118,122]. Yamaguchi, et al. [69] revealed that FSP1positive podocytes in the urine of diabetic patients selectively expressed Snail1 and integrin-linked kinase, a well-known trigger for EMT. The number of FSP1-positive podocytes correlated with the clinical and pathological findings of DN [69].

Furthermore, TGF- $\beta 1$ stimulates the VEGF expression by podocytes, involving in the mechanisms of increased glomerular permeability and glomerulosclerosis formation in DN $[6,118,119]$.

To summarize, TGF- $\beta 1$ exerts multiple effects on podocytes in DM: (i) it reduces the podocytes adhesion to the GBM resulting in podocyturia; (ii) it induces the collagen synthesis by podocytes resulting in GBM thickening (iii) it induces the podocytes EMT resulting in podocytes depletion; (iv) it impairs the architecture of SD and alters the podocytes permselectivity; (v) it increases of podocytes apoptosis resulting in podocytes depletion.

\section{Vascular Endothelial Growth Factor A (VEGF-A)}

VEGF-A is produced in great quantities by the glomerular podocytes. It is important for maintaining of the glomerular filtration barrier integrity and is a survival factor for podocytes, endothelial and mesangial cells [6, 124-126]. Activation of VEGF synthesis in podocytes was shown in vitro and in vivo by high glucose concentrations, AT-II (via ATR1 and ATR2), TGF- $\beta 1$, HIF-1 $\alpha$ (via AT-II and ATR2), AGEs, ROS, insulin) $[6,124,127]$.

VEGF maintains glomerular filtration barrier integrity by signaling through VEGF-receptor 2 (VEGFR2), which is most abundantly expressed in the glomerular endothelium. There are clinical and experimental evidences that in DM glomerular VEGFA expression and urinary levels can be elevated in the early stages of kidney disease [128-130] and significantly decreased in the foci of glomerulosclerosis that appears to be linked to the development of podocytopenia $[6,30,131]$. Interestingly, the induction of podocyte VEGF-A overexpression in adult mice causes glomerular disease with GBM thickening, glomerulomegaly, mesangial proliferation and FP effacement, a phenotype strikingly similar to early DN [132].

VEGF by binding the VEGFR2-nephrin-actin complex activates it and thus regulates the size and form of podocytes $[6,124]$. Experimental data showed that antiapoptotic action of nephrin is associated with its phosphorylation under the influence of VEGF via activation of $\mathrm{Bcl}-2$ protein $[124,133]$. In contrast, reduce of nephrin phosphorylation contributes to podocytes apoptosis via nephrin linking with $\beta$-arrestin-2 [134]. DM violates the autoregulation of VEGF-signalling pathways. Thus, VEGF inhibits the nephrin formation in podocytes, promoting the FP violation. The decrease of VEGF expression impairs the podocytes survival, accelerating the podocytopenia [124]. The evidence has been obtained that VEGF-A stimulates the production of the $\alpha 3$ chain of type IV collagen by podocytes, resulting GBM thickening and violation of its permeability $[6,124]$. It is believed that this synthesis is mediated by VEGF-A via TGF- $\beta 1$ and TGF- $\beta 1$ receptor $[6,124]$.

\section{Reactive oxygen species (ROS)}

Hyperglycemia and RAAS activation trigger the oxidative stress and ROS formation $[13,15,135]$. An important source of ROS production is NOX, which induces superoxide production via oxidation in the mitochondria, inflammation and stress, such ROS are implicated in the pathogenesis of DN [15,135-137]. Nox4 isoform, which is most abundantly expresses in the kidney and localizes within mesangial and tubular cells, podocytes, plays a critical role in oxidative stress in kidney disease $[6,13,136,137]$. Presented above data, mainly experimental, demonstrated ROS-induced actin polymerization resulting in cytoskeleton and FP violations, podocytes detachment, apoptosis and loss. So, in mice with streptozotocin-induced DM, 
the kidney tissue expressed high concentration of NOX and intensively produced ROS [13]. Suppression of renal NOX is a promising strategy against renal damage [135-137]. Antioxidant drugs including apocynin, an inhibitor of the translocation of cytosolic components of NOX, p38 MAPK inhibitor, or tempol, a superoxide dismutase mimetic, also show renoprotective effect in $D M$, resulting in restoration of $\alpha 3$-integrins expression by podocytes, reduction of podocyte apoptosis and detachment, mesangial matrix expansion and diminution of AU/PU $[6,13]$.

\section{Glycation end-products (AGEs)}

AGEs generated from non-enzymatic glycation and oxidation of proteins or lipids. AGEs renal toxicity is mediated by three mechanisms: i) AGEs can deposit in renal tissue (mesangium, endothelial cells, GBM and podocytes) and modify its structure and function; ii) AGEs can be formed in situ, where they exert a profibrotic effect; iii) AGEs can bind to cell receptors $[13,138]$. The podocytes appear to be sensitive targets of AGEs actions as they express the receptor for AGEs (RAGE). So, the in vitro experiments demonstrated that glycated albumin inhibited nephrin expression in cultured podocytes through the RAGE engagement [139]. The harmful AGEs effect on podocytes is also realized through apoptosis activation by increasing of cell cycle inhibitor p27 synthesis $[13,140]$. There is accumulating evidence to suggest the pathophysiological crosstalk between the RAS and AGEs-RAGE axis in DN. AT-II through $\mathrm{AT}_{2}$ receptors activates the expression of RAGE by podocytes $[13,138,140]$. The AGEs-RAGEmediated ROS generation stimulates the production of TGF- $\beta 1$ via MAPK, and NFKBAGEs induces TGF $\beta-1$ overexpression in podocytes.

AGEs inhibit the expression of neuropilin-1, a transmembrane receptor involved in cell adhesion, and reduces $Z O$ expression, and tight junctions in the SD. AGEs also modify the expression of a cytoskeleton protein actin. All these changes result in increased AU $[13,138,140]$.

AGEs-rich culture medium was found to induce podocytes apoptosis via induction of the transcription factor FOXO4 that contributes to podocytes loss. AGEs-RAGE binding in the podocytes upregulates the MCP-1 release leading to inflammation $[13,138,140]$.

At the same time, the blockade of the AGE effects with antiAGE agents prevented DN progression in experimental DM model $[13,138,140]$. Inhibition of the AGE formation and/or the blockade of RAGE-downstream pathways will be a promising therapeutic strategy for DN treatment.

\section{Conclusion}

Our understanding of podocytes biology has improved significantly in the past decade, we have more detailed knowledge of the mediators and signaling pathways that contribute to podocytes injury in diabetes. Despite the treatments with drugs that block the RAAS and combined antihyperglycaemic drugs, there is still a risk for the DN progression. Undoubtedly, a deeper insight into molecular mechanisms of podocytes injury in DM will lead to new therapeutic options for improving the survival of patients with
DN. In addition to the traditional RAAS blockade, the inhibition of AGEs formation and/or the blockade of RAGE-downstream pathways, the suppression of renal NADPH oxidase and MAPK p38, the impact on VEGF and TGF- $\beta 1$ signaling, improvement of autophagy by $\mathrm{mTORC1}$ pathways regulation may be promising therapeutic approaches for DN. Furthermore, new non-invasive methods for detection of podocytopathy based on the evaluation of the specific podocytes biomarkers in the urine are available today and need to be put into common clinical practice. These urinary tests have good perspectives for early diagnostic and monitoring of DN.

\section{References}

1. IDF (2017) IDF diabetes atlas. Eighth edition.

2. Umanath K, Lewis JB (2018) Update on Diabetic Nephropathy: Core Curriculum. Am J Kidney Dis S0272-6386.

3. Alicic RZ, Rooney MT, Tuttle KR (2017) Diabetic Kidney Disease Challenges, Progress, and Possibilities. Clin J Am Soc Nephrol 12: 2032-2045.

4. Dane MJC, van den Berg BM, Lee DH, Boels MGS, Tiemeier GL, et al. (2015) A microscopic view on the renal endothelial glycocalyx. Am J Physiol Renal Physiol 308: F956-F966.

5. Haneda M, Koya D, Isono M, Kikkawa R (2003) Overview of glucose signaling in mesangial cells in diabetic nephropathy. J Am Soc Nephrol 14:1374-1382.

6. Wolf G, Chen S, Ziyadeh FN (2005) From the periphery of glomerular capillary wall toward the center of disease. Podocyte injury comes of age in diabetic nephropathy. Diabetes 54: 1626-1634.

7. Vallon V, Thomson SC (2012) Renal function in diabetic disease models: the tubular system in the pathophysiology of the diabetic kidney. Annu Rev Physiol 74: 351-375.

8. Haneda M, Koya D, Kikkawa R (2001) Mesangial cell dysfunction as a pathogenesis of diabetic nephropathy. Contrib Nephrol 134: 16-29.

9. Shankland SJ (2006) Podocyte's response to injury: role in proteinuria and sclerosis. Kidney Int 69: 2131-2147.

10. Ziyadeh FN, Wolf G (2008) Pathogenesis of podocytopathy and proteiuria in diabetic glomerulopathy. Cur Diab Rev 4: 39-45.

11. Patrakka J, Tryggvason K (2009) New insights into the role of podocytes in proteinuria. Nat Rev Nephrol 5: 463-468.

12. Reddy GR, Kotlyarevska K, Ransom RF (2008) The podocyte and diabetes mellitus: is the podocyte key to the origins of diabetic nephropathy? Curr Opin Nephrol Hypertens 17: 32-36.

13. Stitt-Cavanagh E, MacLeod L, Kennedy CRJ (2009) The podocyte in diabetic kidney disease. Sci World J 9: 1127-1139.

14. Ding WY, Saleem MA (2012) Current concepts of the podocyte in nephrotic syndrome. Kidney Res Clin Pract 31: 87-93.

15. Sharma V, Sharma P (2013) Role of Different Molecular Pathways in the development of Diabetes Induced Nephropathy. J Diabetes Metab 2013, S9.

16. Wiggins RC (2007) The spectrum of podocytopathies: A unifying view of glomerular diseases. Kidney Int 71: 1205-1214. 
17. Armelloni S, Corbelli A, Giardino L, Li M, Ikehata M, et al. (2014) Podocytes: recent biomolecular developments. BioMol Concepts 4: 319-330.

18. Lewko B, Stepinski J (2009) Hypergliycemia and mechanical stress: Targeting the renal podocyte. J Cell Physiol 221: 288-295.

19. Diez-Sampedro A, Lenz O, Fornoni A (2011) Podocytopathy in diabetes: a metabolic and endocrine disorder. Am J Kidney Dis 58: 637-646.

20. Steffes MW, Schmidt D, McGregory R, Basgen JM (2001) Glomerular cell number in normal subject and in type 1diabetic patients. Kidney 59: 2104-2113.

21. Vestra MD, Masiero A, Roiter AM, Saller A, Crepaldi G, et al. (2003) Is podocyte injury relevant in diabetic nephropathy? Studies in patients with type 2 diabetes. Diabetes 52: 1031-1035.

22. Greka A, Mundel P (2012) Cell biology and pathology of podocytes. Annu Rev Physiol 74: 299-323.

23. Ilatovskaya D, Levchenko V, Lowing A, Staruschenko A, Palygin O (2015) Podocyte injury in diabetic nephropathy: implications of angiotensin II-dependent activation of TRPC channels. Sci Rep 5: 17637.

24. Ilatovskaya D, Palygin O, Levchenko V, Shuyskiy L, Staruschenko A (2017) The role of Angiotensin II in glomerular volume dynamics and podocyte calcium handling. Scientific Reports 7: 299.

25. New LA, Martin CE, Jones N (2014) Advances in slit diaphragm signaling. Curr Opin Nephrol Hypertens 23:420-430.

26. Martin CE, Jones N (2018) Nephrin Signaling in the Podocyte: An Updated View of Signal Regulation at the Slit Diaphragm and Beyond. Front Endocrinol 9: 302.

27. Denhez B, Geraldes P (2017) Regulation of nephrin phosphorylation in diabetes and chronic kidney injury. Adv Exp Med Biol 966: 149-161.

28. New LA, Martin CE, Scott RP, Platt MJ, Keyvani CA, et al. (2016) Nephrin tyrosine phosphorylation is required to stabilize and restore podocyte foot process architecture. J Am Soc Nephrol 27: 2422-2435.

29. Verma R, Venkatareddy M, Kalinowski A, Patel SR, Garg P (2016) Integrin ligation results in nephrin tyrosine phosphorylation in vitro. PLoS One 11: e0148906

30. Brosius FC, Coward RJ (2014) Podocytes, signaling pathways, and vascular factors in diabetic kidney disease. Adv Chronic Kidney Dis 21: 304-310.

31. Luimula P, Ahola H, Wang SX, Aaltonen P, Tikkanen I, et al. (2000) Nephrin in experimental glomerular disease. Kidney Int 58: 1461-1468.

32. Aaltonen $P$, Luimula $P$, Astrom $E$, Palmen $T$, Grönholm $T$, et al. (2001) Changes in the expression of nephrin gene and protein in experimental diabetic nephropathy. Lab Invest 8: 1185-1190.

33. Huh W, Kim DJ, Kim M-K, Kim YG, Oh H-Y, et al. (2002) Expression of nephrin in acquired human glomerular disease. Nephrol Dial Transplant 17: 478-484.

34. NG DPK, Tai BC, Tan E, Leong H, Nurbaya S, et al. (2010). Nephrinuria associates with multiple renal traits in type 2 diabetes. Nephrol Dial Transplant 26: 2508-2514.

35. Doublier S, Salvidio G, Lupia E, Ruotsalainen V, Verzola D, et al. (2003) Nephrin expression is reduced in human diabetic nephropathy of type 1 diabetes. Diabetes 52: 2969-2974.
36. Koop K, Eikhmans $M$, Baelde HJ, Kawachi $H$, De Heer $E$, et al. (2003) Expression of podocyte-associated molecules in acquired human kidney diseases. Am J Soc Nephrol 14: 2063-2071.

37. Jim B, Ghanta M, Qipo A, Fan Y, Chuang PY, et al. (2012) Dysregulated nephrin in diabetic nephropathy of type 2 diabetes: A cross Sectional study. PLoS ONE 7: e36041.

38. Nakatsue T, Koike H, Han GD, Suzuki K, Miyauchi N, et al. (2005) Nephrin and podocin dissociate at the onset of proteinuria in experimental membranous nephropathy. Kidney Int 67: 2239-2253.

39. Patari A, Forsblom C, Havana M, Taipale H, Groop PH, et al. (2003) Nephrinuria in diabetic nephropathy of type 1 diabetes. Diabetes 52: 2969-2974.

40. Chang JH, Paik SY, Mao L (2012) Diabetic kidney disease in FVB/NJ akita mice: temporal pattern of kidney injury and urinary nephrin excretion. PLoS ONE 7(4): e3394.

41. Wang G, Lai FM-M, Lai K-B, Chow K-M, Li K-TP, et al. (2007) Messenger RNA expression of podocyte associated molecules in the urinary sediment of patients with diabetic nephropathy. Nephron-Clinical Practice 106: 169-179.

42. Zheng M, Lv LL, Ni J, Ni HF, Li Q, et al. (2011) Urinary podocyteassociated mRNA profile in various stages of diabetic nephropathy. PLoS One 6: e20431.

43. Wickman L, Afshinnia F, Wang SQ, Yang Y, Wang F, et al. (2013) Urine podocyte mRNAs, proteinuria, and progression in human glomerular diseases. J Am Soc Nephrol 24: 2081-2095.

44. Weil EJ, Lemley KV, Mason CC, Yee B, Jones LI, et al. (2012) Podocyte detachment and reduced glomerular capillary endothelial fenestration promote kidney disease in type 2 diabetic nephropathy. Kidney Intern 82: 1010-1017.

45. Sawada K, Toyoda M, Kaneyama N, Shiraiwa S, Han Miyatake, et al. (2016) Upregulation of $\alpha 3 \beta 1$-Integrin in Podocytes in Early-Stage Diabetic Nephropathy. J Diabetes Res 9265074.

46. Chen HC, Chen CA, Guh JY (2000) Altering expression of alpha3beta1 integrin on podocytes of human and rats with diabetes. Life Sci 67: 2345-2353.

47. Kitsiou PV, Tzinia AK, Stetler-Stevenson WG, MichaelA F, Fan WW et al. (2003) Glucose-induced changes in integrins and matrixrelated functions in cultured human glomerular epithelial cells. Am J Physiol Renal Physiol 284: F671-679.

48. Vogelmann SU, Nelson WJ, Myers BD, Lemley KV (2003) Urinary excretion of podocytes in health and renal disease. Am J Physiol Renal Physiol 285: F40-F48.

49. Nakamura T, Ushiyama C, Suzuki S, Hara M, Shimada N, et al. (2000) Urinary excretion of podocytes in patients with diabetic nephropathy. Nephrol Dial Transplant 15: 1379-1383.

50. Yu D, Petermann A, Kunter U, Rong S, Shankland SJ, et al. (2005) Urinary podocyte loss is a more specific marker of ongoing glomerular damage than proteinuria. JASN 16: 1733-1741.

51. Hara M, Yamagata K, Tomino Y, Saito A, Hirayama Y, et al. (2012) Urinary podocalyxin is an early marker for podocyte injury in patients with diabetes: establishment of a highly sensitive ELISA to detect urinary podocalyxin. Diabetologia 55: 2913-2919.

52. Ye H, Bai X, Gao H, Li Li, Wu C, et al. (2014) Urinary podocalyxin positive element occurs in the early stage of diabetic nephropathy and is correlated with a clinical diagnosis of diabetic nephropathy. J Diabetes Complicat 28: 96-100. 
53. Sato Y, Wharram BL, Lee SK, Wickman L, Goyal M, et al. (2009) Urine podocyte mRNAs mark progression of renal disease. J Am Soc Nephrol 20: 1041-1052.

54. Wang G, Lai FMM, Lai KB, Chow KM, Kwan BCH, et al. (2010) Intrarenal and urinary mRNA expression of podocyte-associated molecules for the estimation of glomerular podocyte loss. Renal Failure 32: 372-379.

55. Lytvyn Y, Xiao F, Kennedy CR, Perkins BA, Reich HN, et al. (2017) Assessment of urinary microparticles in normotensive patients with type 1 diabetes. Diabetologia 60: 581-584.

56. Xu WC, Qian G, Liu AQ, Li YQ, Zou HQ (2018) Urinary Extracellular Vesicle: A Potential Source of Early Diagnostic and Therapeutic Biomarker in Diabetic Kidney Disease. Chin Med J 131: 1357-1364.

57. Böttinger EP (2007) TGF-beta in renal injury and disease. Semin Nephrol 27: 309-320.

58. Schiffer M, Bitzer M, Roberts IS, Kopp JB, Ten Dijke P, et al. (2001) Apoptosis in podocytes induced by TGF-beta and Smad7. J Clin Invest 108: 807-816.

59. Schiffer M, Mundel P, Shaw AS, Bottinger EP (2004) A novel role for the adaptor molecule CD2-associated protein in transforming growth factor-betainduced apoptosis. J Biol Chem 279: 3700437012.

60. Kim YH, Goyal M, Kurnit D, Wharram B, Wiggins J, et al. (2001). Podocyte depletion and glomerulosclerosis have a direct relationship in the PAN-treated rat. Kidney Int 60: 957-968.

61. Susztac K, Raff AC, Schiffer M, Böttinger EP (2006) Glucoseinduced reactive oxygen species cause apoptosis of podocytes and podocyte depletion at the onset of diabetic nephropathy. Diabetes 55: 226-233.

62. Versola D, Gandolfo MT, Ferrario F, Rastaldi MP, Villaggio B, et al. (2007) Apoptosis in the kidney of the patients with type 2 diabetic nephropathy. Intern Soc Nephrol 72: 1262-1272.

63. Sanches-Nino MD, Sanz $A B$, Sanches-Lopes $E$, Ruiz-Ortega $M$, Benito-Martin A, et al. (2012) HSP27/HSPB1 as an adaptive podocyte antiapoptotic protein activated by high glucose and angiotensin II. Lab Invest 92: 32-45.

64. Tagawa A, Yasuda M, Kume S, Yamahara K, Nakazawa J, et al. (2016) Impairment of autophagy by up regulation of mTORC1 is implicated in the initiation and progression of DN. Diabetes 65 755-67.

65. Kitada M, Ogura Y, Monno I, Koya D (2017) Regulating autophagy as a therapeutic target for diabetic nephropathy. Curr Diab Rep 17: 53.

66. Liu Y (2010) New insights into epithelial-mesenchymal transitition contribute in kidney fibrosis. J Am Soc Nephrol 21: 212-222.

67. Herman-Edelstein $M$, Thomas MC, Thallas-Bonke V, Saleem M, Cooper ME (2011). Dedifferentiation of immortalized human podocytes in response to transforming growth factor-beta: a model for diabetic podocytopathy. Diabetes 60: 1779-1788.

68. Derynck R, Muthusamy BP, Saeteurn KY (2014) Signaling pathway cooperation in TGF- $\beta$-induced epithelial-mesenchymal transition. Curr Opin Cell Biol 31: 56-66.

69. Yamaguchi $Y$, Iwano $M$, Suzuki D, Nakatani K, Kimura K, et al. (2009) Epithelial-mesenchymal transition as a potential explanation for podocyte depletionin diabetic nephropathy. Am J Kidney Dis 54: 653-664.
70. Wiggins JE, Goyal M, Sanden SK, Wharram BL, Shedden KA, et al. (2005) Podocyte hypertrophy, "adaptation," and "decompensation" associated with glomerular enlargement and glomerulosclerosis in the aging rat: prevention by calorie restriction. JASN 16: 2953-2966.

71. Petermann AT, Pippin J, Durvasula R, Pichler, Hiromura K, et al. (2005) Mechanical stretch induces podocyte hypertrophy in vitro. Kidney Int 67: 157-166.

72. Kim NH (2005) Podocyte hypertrophy in diabetic nephropathy. Nephrology 10: S14-S16.

73. Wharram B, Goyal M, Wiggins J, Sanden SK, Hussain S, et al. (2005) Podocyte depletion causes glomerulosclerosis. Diphtheria toxin-induced podocyte depletion in rats expressing the human DTR transgene. J Am Soc Nephrol 16: 2941-2952.

74. Steffes MW, Schmidt D, Mc Grery R, Basgen JM (2001) Glomerular cell number in normal subjects and in type 1diabetic patients. Kidney Intern 59: 2104-2113.

75. Meyer TW, Bennett PH, Nelson RG. (1999) Podocyte number predicts long-term urinary albumin excretion in Pima Indians with type II diabetes and microalbuminuria. Diabetologia 42: 1341-1344.

76. White KE, Bilous RW (2004) Structural alterations to the podocyte are related to proteinuria in 2 type diabetic patients. Nephrol Dial Transplant 9: 1437-1440.

77. Wang W, Wang Y, Long J, Wang J, Haudek SB, et al. (2012). Mitochondrial fission triggered by hyperglycemia is mediated by ROCK1 activation in podocytes and endothelial cells. Cell Metab 15: $186-200$

78. Eid AA, Gorin Y, Fagg BM, Maalouf R, Barnes JL, et al. (2009) Mechanisms of podocyte injury in diabetes: role of cytochrome P450 and NADPH oxidases. Diabetes 58: 1201-1211.

79. Liu BC, Song X, Lu XY, Li DT, Eaton DC, et al. (2013) High glucose induces podocyte apoptosis by stimulating TRPC6 via elevation of reactive oxygen species. Biochim Biophys Acta 1833: 1434-1442.

80. Zhang L, Li R, Shi W, Liang X, Liu S. et al. (2013) NFAT2 inhibitor ameliorates diabetic nephropathy and podocyte in jury in $\mathrm{db} / \mathrm{db}$ mice. Br J Pharmacol 170: 426-439.

81. Li R, Zhang L, Shi W, Zhang B, Liang X, et al. (2013). NFAT2 mediates highg glucose-induced glomerular podocyte apoptosis through increased Bax expression. Exp Cell Res 319: 992-1000.

82. Kumar PA, Welsh GI, Saleem MA, Menon RK (2014) Molecular and cellular events mediating glomerular podocyte dysfunction and depletion in diabetes mellitus. Front Endocrinol 5: 151.

83. Coward RJ, Welsh GI, Yang J, Tasman C, Lennon R, et al. (2005) The human glomerular podocyte is a novel target for insulin action. Diabetes 54: 3095-3102.

84. Mima $A$, Ohshiro $Y$, Kitada $M$, Matsumoto $M$, Geraldes $P$, et al (2011) Glomerular specific protein kinase C-beta-induced insulin receptor substrate-1 dysfunction and insulin resistance in rat models of diabetes and obesity. Kidney Int 79: 883-896.

85. Welsh GI, Hale LJ, Eremina V, Jeansson M, Maezawa Y, et al. (2010) Insulin signaling to the glomerular podocyte is critical for normal kidney function. Cell Metab 12: 329-340.

86. Drapeau N, Lizotte F, Denhez B, Guay A, Kennedy CR (2013) Expression of SHP-1 induced by hyperglycemia prevents insulin actions in podocytes. Am J Physiol Endocrinol Metab 304: E1188E1198. 
87. Lennon R, Welsh GI, Singh A,Satchell SC, Coward RJ, et al. (2009) Rosiglitazone enhances glucose uptake in glomerular podocytes using the glucose transporter GLUT1. Diabetologia 52: 1944-52.

88. Ichihara A, Kaneshiro Y, Takemuro T (2007) The (pro)renin receptor and the kidney. Sem Nephrol 27: 524-528.

89. Yoo TH, Li JJ, Kim JJ (2007) Activation of the renin-angiotensin system within podocytes in diabetes. Kidney Int 71: 1019-1027.

90. Sakoda M, Itoh H, Ichihara A (2011) Podocytes as a target of prorenin in diabetes. Current Diabetes Reviews 7: 17-21.

91. Wennmann DO, Hsu H-H, Pavenstadt H (2012) The reninangiotensin-aldosterone system in podocytes. Seminars in Nephrology 32: 377-384.

92. Abbate M, Zoja C, Morigi M (2002) Transforming growth factorbeta 1 is upregulated by podocytes in response to excess intraglomerular passage of proteins: a central pathway in progressive glomerulosclerosis. Am J Pathol 161: 2179-2193.

93. Durvasula RV, Petermann AT, Hiromura K, Blonski M, Pippin J, et al. (2004). Activation of local tissue angiotensin system in podocytes by mechanical strain. Kidney Intern 65: 309.

94. Durvasula RV, Shankland SJ (2008) Activation of local angiotensin system in podocytes by glucose. Am J Physiol Renal Physiol 294: F830-839.

95. Nitschke R, Henger A, Ricken S, Gloy J, Müller V, et al. (2000). Angiotensin II increases the intracellular calcium activity in podocytes of the intact glomerulus. Kidney Int 57: 41-49.

96. Ding G., Reddy K., Kapasi A.A.,Franki N, Gibbons N, et al. (2002) Angiotensin II induces apoptosis in rat glomerular epithelial cells Am J Physiol Renal Physiol; 283: F173-180.

97. Yadav A, Vallabu S, Arora S, Tandon P, Slahan D, et al. (2010) ANG II promotes autophagy in podocytes. Am J Physiol Cell Physiol 299: C488-496.

98. Mifsud SA, Allen TJ, Bertram JF, Hulthen UL, Kelly DJ, et al. (2001) Podocyte foot process broadening in experimental diabetic nephropathy: amelioration with renin-angiotensin blockade. Diabetologia 44: 878-882.

99. Gross ML, El-Shakmak A, Szabo A, Koch A, Kuhlmann A, et al. (2003) ACE-inhibitor but not endothelin receptor blockers prevent podocyte loss in early nephropathy. Diabetologia 46: 856-868.

100. Nijenhuis T, Sloan AJ, Hoenderop JG, Flesche J, van Goor H, et al. (2011) Angiotensin II contributes to podocyte injury by increasing TRPC6 expression via an NFAT-mediated positive feedback signaling pathway. Am J Pathol 179: 1719-1732.

101. Sonneveld R, van der Vlag J, Baltissen MPA, Sjoerd AJ, Verkaart SAJ, et al. (2014) Glucose specifically regulates TRPC6 expression in the podocyte, in an Angll-dependent manner. Am J Pathol 184: $1715-1726$.

102. Chi X, Hu-Bo, Yu SY, Yin-Lianghong, Meng-Yu, et al. (2015) Losartan treating podocyte injury induced by Ang II via downregulation of TRPC6 in podocytes. J Renin Angiotensin Aldosterone Syst 16: 1118-1124.

103. Jia J, Ding G, Zhu J, Chen C, Liang W, et al. (2008) Angiotensin II infusion induces nephrin expression changes and podocyte apoptosis. Am J Nephrol 28: 500-507.

104. Miceli I, Burt D, Tarabra E, Camussi G, Cavallo P, et al. (2010) Stretch reduces nephrin expression via an angiotensin II-AT(1)dependent mechanism in human podocytes: effect of rosiglitazone. Am J Physiol Renal Physiol 298: F381-390.
105. Königshausen E, Zierhut UM, Ruetze M, Potthoff SA, Stegbauer J, et al. (2016) Angiotensin II increases glomerular permeability by $\beta$ arrestin mediated nephrin endocytosis. Sci Rep 6: 39513.

106. Chen S, Lee JS, Iglesias-de la Cruz MC, Wang A, Izquierdo-Lahuerta A, et al. (2005) Angiotensin II stimulates alpha3 (IV) collagen production in mouse podocytes via TGF-beta and VEGF signalling: implications for diabetic glomerulopathy. Nephrol Dial Transplant 20: $1320-1328$.

107. Brinkkoetter PT, Holtgrefe S, van der Woude FJ, Yard BA (2004) Angiotensin II type 1-receptor mediated changes in heparan sulfate proteoglycans in human SV40 transformed podocytes. J Am Soc Nephrol 15: 33-40.

108. Nguyen G, Muller DN (2010) The biology of the (pro)renin receptor. J Am Soc Nephrol 21: 18-23.

109. Nguyen G, Delarue F, Burckle C, Bouzhir L, Giller T, et al. (2002) The renin/prorenin receptor in angiotensin II production and cellular responses to renin. J Clin Invest 109: 1417-1427.

110. Riediger F, Quack I, Qadri F, Hartleben B, Park JK, et al. (2011) Prorenin receptor is essential for podocyte autophagy and survival. J Am Soc Nephrol 22: 2193-2202.

111. Matavelli LC, Huang J, Siragy HM (2010) (Pro)renin receptor contributes to diabetic nephropathy by enhancing renal inflammation. Clin Exp Pharmacol Physiol 37: 277-282.

112. Sakoda M, Ichihara A, Kurauchi-Mito A, Narita T, Kinouchi K, et al. (2010) Aliskiren inhibits intracellular angiotensin II levels without affecting (pro)renin receptor signals in human podocytes. Am J Hypertens 23: 575-580.

113. Phillips LM, Wang Y, Dai T, Feldman DL, Lapage J, et al. (2011) The renin inhibitor aliskiren attenuates high-glucose induced extracellular matrix synthesis and prevents apoptosis in cultured podocytes. Nephron Exp Nephrol 118: e49-59.

114. Shibata S, Nagase M, Yoshida S, Kawachi H, Fujita T (2007) Podocyte as the target for aldosterone: roles of oxidative stress and Sgk1. Hypertension 49: 355-364.

115. Zhu C, Huang S, Yuan Y (2011) Mitochondrial dysfunction mediates aldosterone-induced podocyte damage: a therapeutic target of PPARY. Am J Pathol 178: 2020-2031.

116. Zhu Y, Usui HK, Sharma K (2007) Regulation of transforming growth factor beta in diabetic nephropathy: implications for treatment. Semin Nephrol 27: 153-160.

117. Lee HS (2012) Mechanisms and consequences of TGF- $\beta$ overexpression by podocytes in progressive podocyte disease. Cell Tissue Res 347: 129-140.

118. Lee HS (2013) Pathogenic Role of TGF- $\beta$ in Diabetic Nephropathy. J Diabetes Metab S9: 008.

119. Dessapt C, Baradez MO, Hayward A, Dei Cas A, Thomas SM, et al. (2009) Mechanical force sand TGFbeta1 reduce podocyte adhesion through alpha3beta1 integrin downregulation. Nephrol Dial Transplant 24: 2645-2655.

120. Das R, Xu S, Quan X, Nguyen TT, Kong ID (2014) Upregulation of mitochondrial Nox4 mediates TGF-beta-induced apoptosis in cultured mouse podocytes. Am J Physiol Renal Physiol 306: F155-167.

121. Derynck R, Zhang YE (2003) Smad-dependent and Smadindependent pathways in TGF-beta family signalling. Nature 425: 577-584.

122. Abe Y, Sakairi T, Beeson C, Kopp JB (2013) TGF-beta1 stimulates mitochondrial oxidative phosphorylation and generation of 
reactive oxygen species in cultured mouse podocytes, mediated in the part by the mTOR pathway. Am J Physiol Renal Physiol 305: F1477-1490.

123. Li Y, Kang YS, Dai C, Kiss LP, Wen X, et al. (2008) Epithelial-tomesenchymal transition is a potential pathway leading to podocyte dysfunction and proteinuria. Am J Pathol 172: 299-308.

124. Tufro A, Veron D (2012) VEGF and podocytes in diabetic nephropathy. Sem Nephrol 32: 385-393.

125. Ferrara N, Gerber HP, LeCouter J (2003) The biology of VEGF and its receptors. Nat Med 9: 669-676.

126. Foster RR, Hole R, Anderson K, Satchell SC, Coward RJ, et al. (2003) Functional evidence that vascular endothelial growth factor may act as an autocrine factor on human podocytes. Am J Physiol Renal Physiol 284: F1263-F1273.

127. Hale LJ, Hurcombe J, Lay A, et al. (2013) Insulin directly stimulates VEGFA production in the glomerular podocyte. Am J Physiol Renal Physiol 305: F182-F188.

128. Veron D, Bertuccio CA, Marlier A, Santamaría B, Valverde AM, et al. (2011) Podocyte vascular endothelial growth factor (VEGF164) overexpression causes severe nodular glomerulosclerosis in a mouse model of type 1 diabetes. Diabetologia 54: 1227-1241.

129. Kim NH, Kim KB, Kim DL, Kim SG, Choi KM, et al. (2004) Plasma and urinary vascular endothelial growth factor and diabetic nephropathy in type 2 diabetes mellitus. Diabet Med 21: 545-551.

130. Hohenstein B, Hausknecht B, Boehmer K, Riess R, Brekken RA (2006) Local VEGF activity but not VEGF expression is tightly regulated during diabetic nephropathy in man. Kidney Int 69: 1654-1661.

131. Baelde HJ, Eikmans M, Lappin DW, Doran PP, Hohenadel D, et al. (2007) Reduction of VEGF-A and CTGF expression in diabetic nephropathy is associated with podocyte loss. Kidney Int 71: 637-645.

132. Veron D, Reidy K, Villegas G, Kopp J, Thomas D, Tufro A (2010) Induction of podocyte VEGF-A overexpression in adult mice causes glomerular disease. Kidney Int 77: 989-999.

133. Fan Q, Xing Y, Ding J, Guan N (2009) Reduction in VEGF protein and phosphorylated nephrin associated with proteinuria in adriamycin nephropathy rats. Nephron Exp Nephrol 111: e92.

134. Foster RR, Saleem MA, Mathieson PW, Bates DO, Harper SJ (2005) Vascular endothelial growth factor and nephrin interact and reduce apoptosis in human podocyte. Am J Physiol Renal Physio 288: F48-57.

135. Jha JC, Banal C, Chow BS, Cooper ME, Jandeleit-Dahm K (2016) Diabetes and Kidney Disease: Role of Oxidative Stress. Antioxid Redox Signal 25: 657-684.

136. Gorin Y, Block K (2013) Free Nox4 and diabetic nephropathy: with a friend like this, who needs enemies? Radic Biol Med 61: $130-142$.

137. Gorin Y, Block K (2013) Nox as a target for diabetic complications. Clin Sci 25: 361-382.

138. Daroux M, Grossin M, Boulanger E (2012) AGE, RAGE, and Diabetic Nephropathy. US Endocrinol 8: 98-103.

139. Doublier S, Salvidio G, Lupia E, Ruotsalainen V, Verzola, et al. (2003) Nephrin Expression Is Reduced in Human Diabetic Nephropathy: Evidence for a Distinct Role for Glycated Albumin and Angiotensin II. Diabetes 52: 1023-1030.

140. Yamagishi SI, Matsu T (2010) Advanced glycation end products, oxidative stress and diabetic nephropathy. xid Med Cell Longev 3: 101-108. 